\title{
Contents of Volume 24
}

Factorization of Generalized Theta Functions Revisited

X.T. Sun

Rota-Baxter TD Algebra and Quinquedendriform Algebra

S.Y. Zhou, L. Guo

On Existence of Hall Subgroups in Finite Groups

Y.F. Liu, W.B. Guo, A.N. Skiba

Generalized Graph Ideals of Linear Type

M. La Barbiera, P.L. Staglianò

A Study of the Number of Roots of $x^{k}=g$ in a Finite Group via

Its Frobenius-Schur Indicators

S.K. Prajapati, R. Sarma

Gröbner-Shirshov Basis of Twisted Generic Composition Algebras of Affine Type

G. Yunus, A. Obul

The Inductive Blockwise Alperin Weight Condition for $\operatorname{PSL}(3, q)$

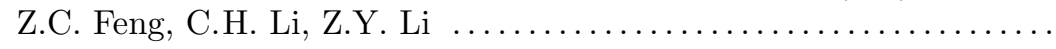

Finite Groups with Automorphism Groups Having Orders $4 p q^{2}$

$(2<p<q)$ II

Y. Zeng, J.B. Li, G.Y. Chen

A System of Periodic Discrete-time Coupled Sylvester Quaternion

Matrix Equations

Z.H. He, Q.W. Wang

The McKay Conjecture for Finite Groups with Abelian Sylow

3-Subgroups

Z.C. Feng, C.H. Li, Z.Y. Li

Structure of Hyperbolic Unitary Groups II: Classification of

E-Normal Subgroups

R. Preusser

A System of Matrix Equations over the Quaternion Algebra with

Applications

X.R. Nie, Q.W. Wang, Y. Zhang

Gradewise Properties of Subgroups and Their Applications

W.B. Guo, A.N. Skiba 
On Free Pre-Lie Algebras

Y. Li, Q.H. Mo

Automorphisms and Verma Modules for Generalized 2-dim

Affine-Virasoro Algebra

W.L. Ruan, H.L. Zhang, J.C. Sun

Presenting Little $q$-Schur Algebras $u_{k}(2, r)$

Z.H. Bian, M.Q. Liu

Jacobson's Lemma via Gröbner-Shirshov Bases

X.G. Zhao

$C_{2}$-Cofiniteness of Cyclic-Orbifold Vertex Operator Superalgebras

C.R. Ai

A New Composition-Diamond Lemma for Dialgebras

G.L. Zhang, Y.Q. Chen

Class-Preserving Coleman Automorphisms of Finite Groups with Prescribed Centralizers

Z.X. Li, H.W. Gao

Quasiresiduals in Semigroups with Natural Partial Order

H. Mitsch

Constructions of $1 \frac{1}{2}$-Designs from Unitary Geometry over Finite

Fields

R.Q. Feng, L.W. Zeng, Y. Zhang

Generalized Derivations in Rings with Involution

N.A. Dar, A.N. Khan

Cohen-Macaulay Lexsegment Complexes in Arbitrary Codimension

H. Haghighi, S. Yassemi, R. Zaare-Nahandi

The Classification of Extensions of $L_{s l_{3}}(k, 0)$

C.R. Ai, X.J. Lin

Automorphisms for Some Symmetric Multiparameter Quantized

Weyl Algebras and Their Localizations

X. Tang

The Projective Cover of the Trivial Representation for a Finite Group of Lie Type in Defining Characteristic

S. Koshitani, J. Müller

Heptavalent Symmetric Graphs of Order $16 p$

S.T. Guo, H.L. Hou, Y. Xu

On Connected Tetravalent Cayley Graphs of a Non-abelian Group of Order $3 p^{2}$

M.R. Darafsheh, M. Abdollahi 
Nil Clean Graphs of Rings

D.K. Basnet, J. Bhattacharyya

Embedding Countably Generated Algebras into Simple

2-Generated Algebras

Q.H. Mo, X.G. Zhao, Q.N. Pan

Property of Almost Cohen-Macaulay over Extension Modules

S. Tabejamaat, A. Mafi, K. Ahmadi Amoli

$q$-Deformations of 3-Lie Algebras

R.P. Bai, L.X. Lin, Y. Zhang, C.C. Kang

Automorphism Groups of a Class of Cubic Cayley Graphs on

Symmetric Groups

X.Y. Huang, Q.X. Huang, L. Lu

Higher Iterated Hilbert Coefficients of the Graded Components of

Bigraded Modules

S.Sh. Arkian

Gröbner-Shirshov Bases for Replicated Algebras

P.S. Kolesnikov

Cotorsion Pairs in $\mathcal{C}_{N}(\mathscr{A})$

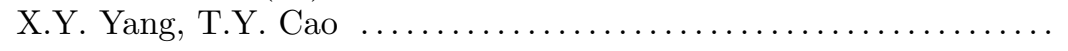

On Baer-Kaplansky Classes of Modules

D. Keskin Tütüncü, R. Tribak

On Generalization of Cycles and Chordality to Clutters from an Algebraic Viewpoint

A. Nikseresht, R. Zaare-Nahandi

Retractable Compact Directed Complete Poset (Acts)

M. Mehdi Ebrahimi, M. Mahmoudi, M. Yavari

Objective Triangle Functors in Adjoint Pairs

P. Zhang, L. Zhu ...

On Formulas and Some Combinatorial Properties of Schubert

Polynomials

Z.R. Zhang, Y.Q. Chen

Local Superderivations on Basic Classical Lie Superalgebras

H.X. Chen, Y. Wang, J.Z. Nan

The Action on $p$-Groups and $p$-Supersolvability

P.F. Bai, X.Y. Guo, B.R. Zhang

Irreducible Weight Modules with a Finite-Dimensional Weight Space over the Twisted $N=1$ Schrödinger-Neveu-Schwarz Algebra 
The Number of Rational Points of a Family of Algebraic Varieties over Finite Fields

S.N. Hu, J.Y. Zhao ................................... 705

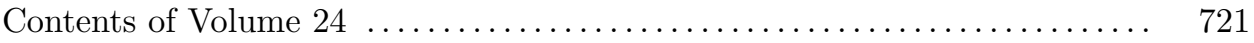

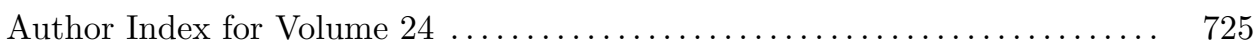

